\title{
PROOF OF SUN'S CONJECTURES ON SUPER CONGRUENCES AND THE DIVISIBILITY OF CERTAIN BINOMIAL SUMS
}

\author{
GUO-SHUAI MAO AND TAO ZHANG
}

ABSTRACT. In this paper, we prove two conjectures of Z.-W. Sun:

$$
2 n\left(\begin{array}{c}
2 n \\
n
\end{array}\right) \mid \sum_{k=0}^{n-1}(3 k+1)\left(\begin{array}{c}
2 k \\
k
\end{array}\right)^{3} 16^{n-1-k} \text { for all } n=2,3, \cdots,
$$

and

$$
\sum_{k=0}^{(p-1) / 2} \frac{3 k+1}{16^{k}}\left(\begin{array}{c}
2 k \\
k
\end{array}\right)^{3} \equiv p+2\left(\frac{-1}{p}\right) p^{3} E_{p-3}\left(\bmod p^{4}\right),
$$

where $p>3$ is a prime and $E_{0}, E_{1}, E_{2}, \cdots$ are Euler numbers.

\section{Introduction}

Let $p>3$ be a prime. A $p$-adic congruence is called a super congruence if it happens to hold modulo some higher power of $p$. Sun [Su1] proved several super congruences involving Euler numbers, such as

$$
\sum_{k=0}^{p-1} \frac{\left(\begin{array}{c}
2 k \\
k
\end{array}\right)}{2^{k}} \equiv(-1)^{(p-1) / 2}-p^{2} E_{p-3}\left(\bmod p^{3}\right) .
$$

Moreover, he proposed many conjectures, such as

Conjecture 1.1. [Su1, Conjecture 5.1] (i) For each $n=2,3, \cdots$ we have

$$
\begin{gathered}
2 n\left(\begin{array}{c}
2 n \\
n
\end{array}\right) \mid \sum_{k=0}^{n-1}(3 k+1)\left(\begin{array}{c}
2 k \\
k
\end{array}\right)^{3} 16^{n-1-k}, \\
2 n\left(\begin{array}{c}
2 n \\
n
\end{array}\right) \mid \sum_{k=0}^{n-1}(42 k+5)\left(\begin{array}{c}
2 k \\
k
\end{array}\right)^{3} 4096^{n-1-k} .
\end{gathered}
$$

Key words and phrases. Central binomial coefficients, congruences, divisibility problem.

2010 Mathematics Subject Classification. 05A10, 11B65, $11 \mathrm{~A} 07$.

This research was supported by the Natural Science Foundation (Grant No. 11571162) of China. 
(ii) Let $p>3$ be a prime. Then

$$
\begin{aligned}
& \sum_{k=0}^{(p-1) / 2} \frac{3 k+1}{16^{k}}\left(\begin{array}{c}
2 k \\
k
\end{array}\right)^{3} \equiv p+2\left(\frac{-1}{p}\right) p^{3} E_{p-3}\left(\bmod p^{4}\right), \\
& \sum_{k=0}^{p-1} \frac{42 k+5}{4096^{k}}\left(\begin{array}{c}
2 k \\
k
\end{array}\right)^{3} \equiv 5 p\left(\frac{-1}{p}\right)-p^{3} E_{p-3}\left(\bmod p^{4}\right),
\end{aligned}
$$

where $(\dot{p})$ denotes the Legendre symbol.

The congruence conjecture (1.4) was solved by $\mathrm{Hu}$ and the first author [HM], while the divisibility conjecture (1.2) remains open.

In [Su2], Z.-W. Sun proved some products and sums divisible by central binomial coefficients, like

$$
4(2 n+1)\left(\begin{array}{c}
2 n \\
n
\end{array}\right) \mid \sum_{k=0}^{n}(4 k+1)\left(\begin{array}{c}
2 k \\
k
\end{array}\right)^{3}(-64)^{n-k}
$$

for any positive integer $n$.

Guo also proved some products and sums divisible by central binomial coefficients. The reader is referred to [G1].

Motivated by the above work, we obtain the following result.

Theorem 1.1. For $n=2,3,4, \ldots$, the assertion (1.1) is true.

Recently, a $q$-analogue of (1.1) has been conjectured by Guo G2, Conjecture 1.7].

Guillera and Zudilin WZ proved the weaker version of the congruence conjecture (1.3)

$$
\sum_{k=0}^{(p-1) / 2} \frac{3 k+1}{16^{k}}\left(\begin{array}{c}
2 k \\
k
\end{array}\right)^{3} \equiv p\left(\bmod p^{3}\right)
$$

using the Wilf-Zeilberger method.

Motivated by their work, we obtain the following result.

Theorem 1.2. Let $p>3$ be a prime. Then the congruence (1.3) is true.

We will prove Theorem 1.1 and 1.2 in Sects. 2 and 3, respectively.

\section{Proof of Theorem 1.1}

Let $p$ be a prime and $n$ a positive integer. Then the $p$-adic evaluation of $n$, denoted by $\operatorname{ord}_{p}(n)$, is the largest number $s$ such that $p^{s} \mid n$. 
Lemma 2.1. For any positive integer $n \geq 6$ and $n \neq 2^{m}+1$, where $m$ is an integer, we have

$$
n-\operatorname{ord}_{2}((n-1) !) \geq 3,
$$

where $\operatorname{ord}_{2}((n-1) !)=\sum_{i=1}^{\infty}\left\lfloor\frac{n-1}{2^{i}}\right\rfloor$.

Proof. Recall the following theorem of Kummer (1852),

The p-adic valuation of the binomial coefficient $\left(\begin{array}{c}m+n \\ m\end{array}\right)$ is equal to the number of 'carry-overs' when performing the addition of $n$ and $m$, written in base $p$.

Noting that $n\left(\begin{array}{c}2 n \\ n\end{array}\right)=\frac{2^{n}(2 n-1) ! !}{(n-1) !}$, we get

$$
\operatorname{ord}_{2}\left(n\left(\begin{array}{c}
2 n \\
n
\end{array}\right)\right)=n-\operatorname{ord}_{2}((n-1) !) .
$$

Hence, it suffices to show that $8 \mid n\left(\begin{array}{c}2 n \\ n\end{array}\right)$.

Write $n=a_{1} a_{2} \cdots a_{k}$ in binary expansion with $a_{1}=1$. Since $n \geq 6$, we have $k>2$.

Case 1. If $a_{k}=a_{k-1}=0$, then $4 \mid n$. Since $2 \mid\left(\begin{array}{c}2 n \\ n\end{array}\right)$ in any case, we get $8 \mid n\left(\begin{array}{c}2 n \\ n\end{array}\right)$.

Case 2. If $a_{k}=0, a_{k-1}=1$, then $2 \mid n$. Since $a_{1}=1$ and $k>2$, by Kummer's Theorem, we have $4 \mid\left(\begin{array}{c}2 n \\ n\end{array}\right)$. Therefore, $8 \mid n\left(\begin{array}{c}2 n \\ n\end{array}\right)$.

Case 3. We have $a_{k}=1$. Since $n \neq 2^{m}+1$ and $k>2$, there must be an integer $i \in\{2,3, \ldots, k-1\}$ such that $a_{i}=1$. By Kummer's Theorem, $8 \mid n\left(\begin{array}{c}2 n \\ n\end{array}\right)$.

Remark 2.1. From Case 3 in the proof, we see that if $n=2^{m}+1 \geq 6$ for some $m$, then

$$
n-\operatorname{ord}_{2}((n-1) !) \geq 2 .
$$

Lemma 2.2. Let $n=2^{m}+1 \geq 6$ be an integer, where $m$ is an integer.

Then we have

$$
8 \mid\left(\begin{array}{c}
4 n-2 \\
2 n-1
\end{array}\right) \pm 2\left(\begin{array}{c}
2 n-2 \\
n-1
\end{array}\right) .
$$

Proof. First, we know

$$
\begin{aligned}
\left(\begin{array}{c}
4 n-2 \\
2 n-1
\end{array}\right) & =\frac{(4 n-2)(4 n-3) \cdots(2 n+1)(2 n) !}{(2 n-1)^{2}(2 n-2)^{2} \cdots(n+1)^{2}(n !)^{2}}=\frac{2^{n}(4 n-3) ! !}{(2 n-1) ! !(n-1) !} \\
& =\frac{2^{n}(4 n-3)(4 n-5) \cdots(2 n+1)}{(n-1) !},
\end{aligned}
$$


hence,

$$
\begin{aligned}
& \left(\begin{array}{c}
4 n-2 \\
2 n-1
\end{array}\right) \pm 2\left(\begin{array}{c}
2 n-2 \\
n-1
\end{array}\right) \\
= & \frac{2^{n}(4 n-3)(4 n-5) \cdots(2 n+1)}{(n-1) !} \pm \frac{2^{n}(2 n-3) ! !}{(n-1) !} \\
= & \frac{2^{n}}{(n-1) !}((4 n-3)(4 n-5) \cdots(2 n+1) \pm(2 n-3) ! !) .
\end{aligned}
$$

Noting that $(4 n-3)(4 n-5) \cdots(2 n+1) \pm(2 n-3) ! ! \equiv 0(\bmod 2)$, we can deduce that

$\operatorname{ord}_{2}\left(\left(\begin{array}{c}4 n-2 \\ 2 n-1\end{array}\right) \pm 2\left(\begin{array}{c}2 n-2 \\ n-1\end{array}\right)\right) \geq n+1-\operatorname{ord}_{2}((n-1) !)=n+1-\operatorname{ord}_{2}(n !)$

since $n=2^{m}+1$ is an odd integer. While $n \geq 6$ and $n=2^{m}+1$ we have

$$
\begin{aligned}
& n+1-\operatorname{ord}_{2}(n !)=2^{m}+2-\sum_{i=1}^{\infty}\left\lfloor\frac{2^{m}+1}{2^{i}}\right\rfloor \\
& =2^{m}+2-\left(2^{m-1}+2^{m-2}+\cdots+2+1\right)=2^{m}+2-\left(2^{m}-1\right)=3 .
\end{aligned}
$$

Therefore, $\operatorname{ord}_{2}\left(\left(\begin{array}{c}4 n-2 \\ 2 n-1\end{array}\right) \pm 2\left(\begin{array}{c}2 n-2 \\ n-1\end{array}\right)\right) \geq 3$, i.e. $8 \mid\left(\begin{array}{c}4 n-2 \\ 2 n-1\end{array}\right) \pm 2\left(\begin{array}{c}2 n-2 \\ n-1\end{array}\right)$. We finish the proof of Lemma 2.2.

Proof of Theorem 1.1. By [MS, Lemma 3.2], for any integer $n \geq 2$,

$$
\sum_{k=0}^{n-1}\left(\begin{array}{c}
n-1 \\
k
\end{array}\right)^{2}\left(\begin{array}{c}
x+k \\
2 n-1
\end{array}\right)=\frac{1}{(4 n-2)\left(\begin{array}{c}
2 n-2 \\
n-1
\end{array}\right)} \sum_{k=0}^{n-1}(2 x-3 k)\left(\begin{array}{l}
x \\
k
\end{array}\right)^{2}\left(\begin{array}{c}
2 k \\
k
\end{array}\right),
$$

set $x=-\frac{1}{2}$ in this combinatorial identity, we have

$$
\begin{aligned}
\sum_{k=0}^{n-1}\left(\begin{array}{c}
n-1 \\
k
\end{array}\right)^{2}\left(\begin{array}{c}
-\frac{1}{2}+k \\
2 n-1
\end{array}\right) & =\frac{1}{(4 n-2)\left(\begin{array}{c}
2 n-2 \\
n-1
\end{array}\right)} \sum_{k=0}^{n-1}(-1-3 k)\left(\begin{array}{c}
-\frac{1}{2} \\
k
\end{array}\right)^{2}\left(\begin{array}{c}
2 k \\
k
\end{array}\right) \\
& =\frac{-1}{n\left(\begin{array}{c}
2 n \\
n
\end{array}\right)} \sum_{k=0}^{n-1}(3 k+1) \frac{\left(\begin{array}{c}
2 k \\
k
\end{array}\right)^{3}}{16^{k}} .
\end{aligned}
$$

It follows that

$$
\begin{aligned}
\frac{\sum_{k=0}^{n-1}(3 k+1)\left(\begin{array}{c}
2 k \\
k
\end{array}\right)^{3} 16^{n-1-k}}{2 n\left(\begin{array}{c}
2 n \\
n
\end{array}\right)} & =-\frac{16^{n-1}}{2} \sum_{k=0}^{n-1}\left(\begin{array}{c}
n-1 \\
k
\end{array}\right)^{2}\left(\begin{array}{c}
-\frac{1}{2}+k \\
2 n-1
\end{array}\right) \\
& =\frac{1}{8} \sum_{k=0}^{n-1}\left(\begin{array}{c}
n-1 \\
k
\end{array}\right)^{2} \frac{(-1)^{k}(2 k) !(4 n-2 k-2) !}{k !(2 n-1) !(2 n-k-1) !} .
\end{aligned}
$$


Therefore, to prove Theorem 1.1, we just need to show that

$$
\frac{1}{8} \sum_{k=0}^{n-1}\left(\begin{array}{c}
n-1 \\
k
\end{array}\right)^{2} \frac{(-1)^{k}(2 k) !(4 n-2 k-2) !}{k !(2 n-1) !(2 n-k-1) !} \in \mathbb{Z} .
$$

When $n=2,3,4,5$, it is easy to check that (2.1) holds. From now on, we can assume $n \geq 6$. For convenience, let

$$
a(n, k)=\frac{(-1)^{k}(2 k) !(4 n-2 k-2) !}{k !(2 n-1) !(2 n-k-1) !} .
$$

For any real numbers $x$ and $y$, we have

$$
\lfloor 2 x\rfloor+\lfloor 2 y\rfloor \geq\lfloor x\rfloor+\lfloor y\rfloor+\lfloor x+y\rfloor .
$$

It follows that, for any prime $p$, we have

$$
\begin{aligned}
\operatorname{ord}_{p}(a(n, k))= & \sum_{i=1}^{\infty}\left(\left\lfloor\frac{2 k}{p^{i}}\right\rfloor+\left\lfloor\frac{4 n-2 k-2}{p^{i}}\right\rfloor-\left\lfloor\frac{k}{p^{i}}\right\rfloor\right. \\
& \left.-\left\lfloor\frac{2 n-1}{p^{i}}\right\rfloor-\left\lfloor\frac{2 n-k-1}{p^{i}}\right\rfloor\right) \\
\geq & 0,
\end{aligned}
$$

i.e. $a(n, k) \in \mathbb{Z}$.

Noting that

$$
a(n, k)=\frac{(-1)^{k} 2^{n}(2 k-1) ! !(4 n-2 k-3) ! !}{(2 n-1) ! !(n-1) !} .
$$

Hence,

$$
\operatorname{ord}_{2}(a(n, k))=n-\operatorname{ord}_{2}((n-1) !) .
$$

If $n \neq 2^{m}+1$, by Lemma 2.1 we have $8 \mid a(n, k)$.

If $n=2^{m}+1$, then for $1 \leq k \leq n-2$, we have $2 \mid\left(\begin{array}{c}n-1 \\ k\end{array}\right)$ and, by Remark 2.1, $4 \mid a(n, k)$.

Hence, $8 \mid\left(\begin{array}{c}n-1 \\ k\end{array}\right)^{2} a(n, k)$. For $k=0$ and $k=n-1$, note that

$$
\left(\begin{array}{c}
n-1 \\
0
\end{array}\right)^{2} a(n, 0)+\left(\begin{array}{c}
n-1 \\
n-1
\end{array}\right)^{2} a(n, n-1)=\left(\begin{array}{c}
4 n-2 \\
2 n-1
\end{array}\right) \pm 2\left(\begin{array}{c}
2 n-2 \\
n-1
\end{array}\right)
$$

which is divisible by 8 according to Lemma 2.2 .

Therefore, for any integer $n \geq 6$, we have

$$
\frac{1}{8} \sum_{k=0}^{n-1}\left(\begin{array}{c}
n-1 \\
k
\end{array}\right)^{2} \frac{(-1)^{k+1}(2 k) !(4 n-2 k-2) !}{k !(2 n-1) !(2 n-k-1) !} \in \mathbb{Z},
$$

which completes the proof of Theorem 1.1. 


\section{Proof of Theorem 1.2}

Lemma 3.1. Let $p>3$ be a prime. For any $0<k \leq(p-1) / 2$, we have

$\frac{1}{p}\left(\begin{array}{c}p-1+2 k \\ (p-1) / 2+k\end{array}\right) \equiv\left(\frac{-1}{p}\right) 4^{p-1} \frac{4^{2 k}}{2 k\left(\begin{array}{c}2 k \\ k\end{array}\right)}\left(1-p\left(H_{2 k-1}-H_{k-1}\right)\right)\left(\bmod p^{2}\right)$.

In particular,

$$
\frac{1}{p}\left(\begin{array}{c}
p-1+2 k \\
(p-1) / 2+k
\end{array}\right) \equiv\left(\frac{-1}{p}\right) \frac{4^{2 k}}{2 k\left(\begin{array}{c}
2 k \\
k
\end{array}\right)}(\bmod p) .
$$

Proof. Recall that Morley $\mathrm{M}$ ] proved that

$$
\left(\begin{array}{c}
p-1 \\
(p-1) / 2
\end{array}\right) \equiv\left(\frac{-1}{p}\right) 4^{p-1}\left(\bmod p^{3}\right) .
$$

for any prime $p>3$. Hence,

$$
\begin{aligned}
\frac{1}{p}\left(\begin{array}{c}
p-1+2 k \\
(p-1) / 2+k
\end{array}\right) & =\left(\begin{array}{c}
p-1 \\
(p-1) / 2
\end{array}\right) \frac{(p+1) \cdots(p+2 k-1)}{\left(\prod_{j=1}^{k}(p+2 j-1) / 2\right)^{2}} \\
& \equiv\left(\frac{-1}{p}\right) 4^{p-1} \frac{(2 k-1) !\left(1+p H_{2 k-1}\right) 2^{2 k}}{\prod_{j=1}^{k}(p+2 j-1)^{2}} \\
& \equiv\left(\frac{-1}{p}\right) 4^{p-1} \frac{(2 k-1) !\left(1+p H_{2 k-1}\right) 2^{2 k}}{\prod_{j=1}^{k}\left((2 j-1)^{2}+2 p(2 j-1)\right)} \\
& \equiv\left(\frac{-1}{p}\right) 4^{p-1} \frac{(2 k-1) !\left(1+p H_{2 k-1}\right) 2^{2 k}}{((2 k-1) ! !)^{2}\left(1+2 p \sum_{j=1}^{k} 1 /(2 j-1)\right)} \\
& =\left(\frac{-1}{p}\right) \frac{4^{p-1+2 k}\left(1+p H_{2 k-1}\right)}{2 k\left(\begin{array}{c}
2 k \\
k
\end{array}\right)\left(1+2 p\left(H_{2 k-1}-H_{k-1} / 2\right)\right)}\left(\bmod p^{2}\right) .
\end{aligned}
$$

Noting that

$$
\begin{aligned}
\frac{1}{1+2 p\left(H_{2 k-1}-H_{k-1} / 2\right)} & =\frac{1-2 p\left(H_{2 k-1}-H_{k-1} / 2\right)}{1-4 p^{2}\left(H_{2 k-1}-H_{k-1} / 2\right)^{2}} \\
& \equiv 1-2 p\left(H_{2 k-1}-H_{k-1} / 2\right)\left(\bmod p^{2}\right) .
\end{aligned}
$$

Hence,

$$
\begin{aligned}
\frac{1}{p}\left(\begin{array}{c}
p-1+2 k \\
(p-1) / 2+k
\end{array}\right) & \equiv\left(\frac{-1}{p}\right) \frac{4^{p-1+2 k}}{2 k\left(\begin{array}{c}
2 k \\
k
\end{array}\right)}\left(1+p H_{2 k-1}\right)\left(1-2 p\left(H_{2 k-1}-H_{k-1} / 2\right)\right) \\
& \equiv\left(\frac{-1}{p}\right) \frac{4^{p-1+2 k}}{2 k\left(\begin{array}{c}
2 k \\
k
\end{array}\right)}\left(1-p\left(H_{2 k-1}-H_{k-1}\right)\right)\left(\bmod p^{2}\right),
\end{aligned}
$$


as desired. The last statement follows immediately from Fermat's Little Theorem.

Lemma 3.2. Let $p>3$ be a prime. For any $0<k \leq(p-1) / 2$, we have

$$
\frac{1}{p}\left(\begin{array}{c}
p-1+2 k \\
2 k
\end{array}\right) \equiv \frac{1}{2 k}\left(1+p H_{2 k-1}\right)\left(\bmod p^{2}\right) .
$$

In particular,

$$
\frac{1}{p}\left(\begin{array}{c}
p-1+2 k \\
2 k
\end{array}\right) \equiv \frac{1}{2 k}(\bmod p)
$$

Proof. Expanding the LHS, we have

$$
\begin{aligned}
\frac{1}{p}\left(\begin{array}{c}
p-1+2 k \\
2 k
\end{array}\right)= & \frac{(2 k+1) \cdots(p-1)}{1 \cdots(p-2 k-1)} \cdot \frac{1}{p-2 k} \cdot \frac{(p+1) \cdots(p+2 k-2)}{(p-2 k+1) \cdots(p-1)} \\
& \cdot \frac{1}{2 k} \prod_{j=1}^{p-2 k-1}(1-p / j) \cdot \frac{1}{1-p / 2 k} \cdot \prod_{j=1}^{2 k-1} \frac{1+p / j}{1-p / j} \\
& \equiv \frac{1}{2 k}\left(1-p H_{p-1-2 k}\right)(1+p / 2 k)\left(1+2 p H_{2 k-1}\right) \\
& \equiv \frac{1}{2 k}\left(1-p H_{p-1-2 k}+p / 2 k+2 p H_{2 k-1}\right)\left(\bmod p^{2}\right) .
\end{aligned}
$$

Recall that Wolstenholem [W] proved that for any prime $p>3$,

$$
H_{p-1} \equiv 0\left(\bmod p^{2}\right) \text {. }
$$

If follows that

$$
\begin{aligned}
H_{p-2 k-1} & \equiv-H_{p-1}+H_{p-2 k-1}=-\sum_{j=1}^{2 k} 1 /(p-j) \\
& \equiv-\sum_{j=1}^{2 k} 1 /(-j)=H_{2 k}(\bmod p) .
\end{aligned}
$$

Therefore,

$$
\frac{1}{p}\left(\begin{array}{c}
p-1+2 k \\
2 k
\end{array}\right) \equiv \frac{1}{2 k}\left(1+p H_{2 k-1}\right)\left(\bmod p^{2}\right) .
$$

Lemma 3.3. Let $p>3$ be a prime. Then

$$
\sum_{k=1}^{(p-1) / 2} \frac{4^{2 k}}{k^{2}\left(\begin{array}{c}
2 k \\
k
\end{array}\right)^{2}} \equiv(-1)^{(p-1) / 2} \frac{3}{p} 4^{1-p} \sum_{k=1}^{(p-1) / 2} \frac{\left(\begin{array}{c}
2 k \\
k
\end{array}\right)}{k}(\bmod p) .
$$


Proof. Noting that

$$
\left(\begin{array}{c}
(p-1) / 2 \\
k
\end{array}\right) \equiv\left(\begin{array}{c}
-1 / 2 \\
k
\end{array}\right)=\frac{\left(\begin{array}{c}
2 k \\
k
\end{array}\right)}{(-4)^{k}}(\bmod p),
$$

we have

$$
\sum_{k=1}^{(p-1) / 2} \frac{4^{2 k}}{k^{2}\left(\begin{array}{c}
2 k \\
k
\end{array}\right)^{2}}=\sum_{k=1}^{(p-1) / 2} \frac{(-4)^{2 k}}{k^{2}\left(\begin{array}{c}
2 k \\
k
\end{array}\right)^{2}} \equiv \sum_{k=1}^{(p-1) / 2} \frac{1}{k^{2}\left(\begin{array}{c}
(p-1) / 2 \\
k
\end{array}\right)^{2}}(\bmod p) .
$$

Recall that Staver [S] proved that

$$
\sum_{k=1}^{n} \frac{\left(\begin{array}{c}
2 k \\
k
\end{array}\right)}{k}=\frac{n+1}{3}\left(\begin{array}{c}
2 n+1 \\
n
\end{array}\right) \sum_{k=1}^{n} \frac{1}{k^{2}\left(\begin{array}{l}
n \\
k
\end{array}\right)^{2}}, \forall n \in \mathbb{Z}^{+} .
$$

Therefore

$$
\begin{aligned}
\sum_{k=1}^{(p-1) / 2} \frac{4^{2 k}}{k^{2}\left(\begin{array}{c}
2 k \\
k
\end{array}\right)^{2}} & \equiv \frac{3}{\frac{p+1}{2}\left(\begin{array}{c}
p \\
(p-1) / 2)
\end{array}\right)} \sum_{k=1}^{(p-1) / 2} \frac{\left(\begin{array}{c}
2 k \\
k
\end{array}\right)}{k} \\
& =\frac{3}{\frac{p+1}{2} \frac{p}{(p+1) / 2}\left(\begin{array}{c}
p-1 \\
(p-1) / 2
\end{array}\right)} \sum_{k=1}^{(p-1) / 2} \frac{\left(\begin{array}{c}
2 k \\
k
\end{array}\right)}{k} \\
& \equiv(-1)^{(p-1) / 2} \frac{3}{p} 4^{1-p} \sum_{k=1}^{(p-1) / 2} \frac{\left(\begin{array}{c}
2 k \\
k
\end{array}\right)}{k}(\bmod p)
\end{aligned}
$$

where we use Morley congruence (3.3) in the last step.

Proof of Theorem 1.2. Take the same WZ pair $F(k, j)$ and $G(k, j)$ as in [WZ],

$$
\begin{aligned}
& F(k, j)=\frac{2 k+2 j+1}{16^{k}}\left(\begin{array}{c}
2 k \\
k
\end{array}\right)^{2} \frac{\left(\begin{array}{c}
2 k+2 j \\
k+j
\end{array}\right)\left(\begin{array}{c}
2 k+2 j \\
2 j
\end{array}\right)}{\left(\begin{array}{c}
2 j \\
j
\end{array}\right)}, \\
& G(k, j)=-\frac{2(2 k-1)}{16^{k-1}}\left(\begin{array}{c}
2 k-2 \\
k-1
\end{array}\right)^{2} \frac{\left(\begin{array}{c}
2 k+2 j-2 \\
k+j-1
\end{array}\right)\left(\begin{array}{c}
2 k+2 j-2 \\
2 j
\end{array}\right)}{\left(\begin{array}{c}
2 j \\
j
\end{array}\right)} .
\end{aligned}
$$

We know that $F(k, j)$ and $G(k, j)$ have the following relation,

$$
F(k, j-1)-F(k, j)=G(k+1, j)-G(k, j) .
$$

Summing up the above equation for $k$ from 0 to $(p-1) / 2$, and then for $j$ from 1 to $(p-1) / 2$, we get

$$
\sum_{k=0}^{(p-1) / 2}(F(k, 0)-F(k,(p-1) / 2))=\sum_{j=1}^{(p-1) / 2}(G((p+1) / 2, j)-G(0, j)) .
$$


Noting that $G(0, j)=0$ and

$$
\sum_{k=0}^{(p-1) / 2} \frac{3 k+1}{16^{k}}\left(\begin{array}{c}
2 k \\
k
\end{array}\right)^{3}=\sum_{k=0}^{(p-1) / 2} F(k, 0)
$$

we have

$$
\sum_{k=0}^{(p-1) / 2} \frac{3 k+1}{16^{k}}\left(\begin{array}{c}
2 k \\
k
\end{array}\right)^{3}=\sum_{k=0}^{(p-1) / 2} F(k,(p-1) / 2)+\sum_{j=1}^{(p-1) / 2} G((p+1) / 2, j) .
$$

Hence, it suffices to determine

$$
\sum_{k=0}^{(p-1) / 2} F(k,(p-1) / 2) \text { and } \sum_{j=1}^{(p-1) / 2} G((p+1) / 2, j)\left(\bmod p^{4}\right) .
$$

First, let us consider

$$
\begin{aligned}
G((p+1) / 2, j) & =-\frac{2 p}{4^{p-1}}\left(\begin{array}{c}
p-1 \\
(p-1) / 2
\end{array}\right)^{2} \frac{\left(\begin{array}{c}
p-1+2 j \\
(p-1) / 2+j
\end{array}\right)\left(\begin{array}{c}
p-1+2 j \\
2 j
\end{array}\right)}{\left(\begin{array}{c}
2 j \\
j
\end{array}\right)} \\
& =-\frac{2 p^{3}}{4^{p-1}} \cdot \frac{\left(\begin{array}{c}
p-1 \\
(p-1) / 2
\end{array}\right)}{\left(\begin{array}{c}
2 j \\
j
\end{array}\right)} \cdot \frac{\left(\begin{array}{c}
p-1+2 j \\
(p-1) / 2+j
\end{array}\right)}{p} \cdot \frac{\left(\begin{array}{c}
p-1+2 j \\
2 j
\end{array}\right)}{p} .
\end{aligned}
$$

By (3.2), (3.3), (3.5) and (3.6), we get

$$
\sum_{j=1}^{(p-1) / 2} G((p+1) / 2, j) \equiv-\frac{3}{2} p^{2} \sum_{j=1}^{(p-1) / 2} \frac{\left(\begin{array}{c}
2 j \\
j
\end{array}\right)}{j}\left(\bmod p^{4}\right)
$$

Now, let us consider

$$
F(k,(p-1) / 2)=\frac{3 k+p}{16^{k}}\left(\begin{array}{c}
2 k \\
k
\end{array}\right)^{2} \frac{\left(\begin{array}{c}
2 k+p-1 \\
k+(p-1) / 2
\end{array}\right)\left(\begin{array}{c}
2 k+p-1 \\
2 k
\end{array}\right)}{\left(\begin{array}{c}
p-1 \\
(p-1) / 2
\end{array}\right)} .
$$


By (3.1), (3.2), (3.3), (3.4), (3.5) and (3.6), we get

$$
\begin{aligned}
& \sum_{k=0}^{(p-1) / 2} F\left(k, \frac{p-1}{2}\right) \\
& =p+\sum_{k=1}^{(p-1) / 2} \frac{p}{16^{k}}\left(\begin{array}{c}
2 k \\
k
\end{array}\right) \frac{2\left(\begin{array}{c}
2 k+p-1 \\
k+(p-1) / 2
\end{array}\right)\left(\begin{array}{c}
2 k+p-1 \\
2 k
\end{array}\right)}{\left(\begin{array}{c}
p-1 \\
(p-1) / 2
\end{array}\right)} \\
& +\sum_{k=1}^{(p-1) / 2} \frac{3 k}{16^{k}}\left(\begin{array}{c}
2 k \\
k
\end{array}\right) \frac{2}{\left(\begin{array}{c}
2 k+p-1 \\
k+(p-1) / 2
\end{array}\right)\left(\begin{array}{c}
2 k+p-1 \\
2 k
\end{array}\right)} \\
& \equiv p+\frac{p^{p-1}\left(\begin{array}{c}
(p-1) / 2 \\
)
\end{array}\right.}{4} \sum_{k=1}^{(p-1) / 2} \frac{\left(\begin{array}{c}
2 k \\
k
\end{array}\right)}{k^{2}}+\frac{3 p^{2}}{4} \sum_{\substack{(p-1) / 2 \\
k=1}}^{\left(\begin{array}{c}
2 k \\
k
\end{array}\right)} \frac{p_{k}}{k}\left(1-p H_{2 k-1}+p H_{k-1}\right)\left(1+p H_{2 k-1}\right) \\
& \equiv p+\frac{p^{3}}{4} \sum_{k=1}^{(p-1) / 2} \frac{\left(\begin{array}{c}
2 k \\
k
\end{array}\right)}{k^{2}}+\frac{3 p^{2}}{4} \sum_{k=1}^{(p-1) / 2} \frac{\left(\begin{array}{c}
2 k \\
k
\end{array}\right)}{k}\left(1+p H_{k-1}\right)\left(\bmod p^{4}\right) .
\end{aligned}
$$

Combining them together, we have

$$
\begin{aligned}
\sum_{k=0}^{(p-1) / 2} \frac{3 k+1}{16^{k}}\left(\begin{array}{c}
2 k \\
k
\end{array}\right)^{3} & \equiv p-\frac{3}{4} p^{2} \sum_{k=1}^{(p-1) / 2} \frac{\left(\begin{array}{c}
2 k \\
k
\end{array}\right)}{k}-\frac{1}{2} p^{3} \sum_{k=1}^{(p-1) / 2} \frac{\left(\begin{array}{c}
2 k \\
k
\end{array}\right)}{k^{2}} \\
& +\frac{3}{4} p^{3} \sum_{k=1}^{(p-1) / 2} \frac{\left(\begin{array}{c}
2 k \\
k
\end{array}\right)}{k} H_{k}\left(\bmod p^{4}\right) .
\end{aligned}
$$

Finally, applying the congruence ([Su1, (1.2)])

$$
\sum_{k=1}^{(p-1) / 2} \frac{\left(\begin{array}{c}
2 k \\
k
\end{array}\right)}{k} \equiv(-1)^{(p+1) / 2} \frac{8}{3} p E_{p-3}\left(\bmod p^{2}\right),
$$

and the congruence $(\underline{\mathrm{MS}},(2.10)])$

$$
\sum_{k=1}^{(p-1) / 2} \frac{\left(\begin{array}{c}
2 k \\
k
\end{array}\right)}{k} H_{k} \equiv \frac{2}{3} \sum_{k=1}^{(p-1) / 2} \frac{\left(\begin{array}{c}
2 k \\
k
\end{array}\right)}{k^{2}}(\bmod p) .
$$

we get the desired result.

Acknowledgment. The authors would like to thank Professor Z.-W. Sun for helpful comments.

\section{REFERENCES}

[WZ] J. Guillera and W. Zudilin, "Divergent" Ramanujan-type supercongruences, Amer. Maath. Soc., 14(3) (2012), 765-777, .

[G1] V.J.W. Guo, Proof of Sun's conjecture on the divisibility of certain binomial sums, Electron. J. Combin. 20(4) (2013). 
[G2] V.J.W. Guo, q-Analogue of two "divergent" Ramanujan-type supercongruences, accepted by Ramanujan Journal.

[HM] D.-W. Hu and G.-S. Mao, On an extension of a Van Hamme supercongruence, Ramanujan J, 42(3) (2017), 713-723.

[MS] G.-S. Mao and Z.-W. Sun, Two congruences involving harmonic numbers with applications, Int. J. Number Theory, 12 (2016), no.02, 527-539.

[M] F. Morley, Note on the congruence $2^{4 n} \equiv(-1)^{2}(2 n) ! /(n !)^{2}$, where $2 n+1$ is a prime, Ann. Math., 9 (1895), 168-170.

[S] T.B. Staver, Om summasjon av potenser av binomialkoeffisienten, Norsk Mat. Tidsskrift, 29 (1947), 97-103.

[Su1] Z.-W. Sun, Super congruences and Euler numbers, Sci. China Math. 54 (2011), no.12, 2509-2535.

[Su2] Z.-W. Sun, Products and sums divisible by central binomial coefficients, Electron. J. Combin. 20 (2013), no.1, 1-14.

[W] J. Wolstenholme, On certain properties of prime numbers, Quart. J. Math., 5 (1862), 35-39.

(Guo-Shuai MaO) Department of Mathematics, Nanjing University, Nanjing 210093, People's Republic of China

E-mail address: mg1421007@smail.nju.edu.cn

(Tao Zhang) Department of Mathematics, University of Maryland, College Park, MD 20742, USA

E-mail address: taozhang@math.umd.edu 